\title{
منهجية بناء علم الاقتصياد الإسلامي: أهمية المراجعة والتقويم
}

\author{
عزالدين مالك الطيب محمد \\ أستاذ الاقتصاد والاقتصاد الإسلامي \\ جامعة أم درمان الإسلامية - السبودان
}

المستخلص. تقدم الورقة إجابات مختصرة ومركزة عن التساؤلات المقدمة من هيئة تحرير

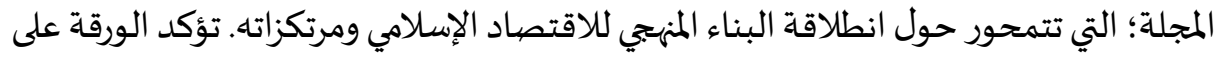

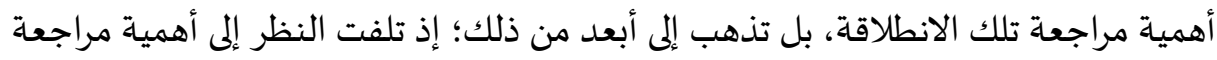

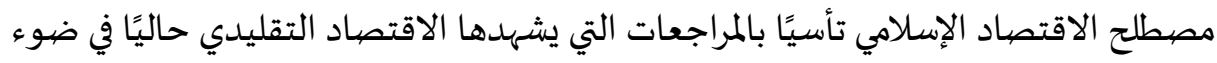

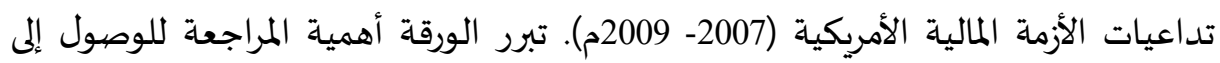

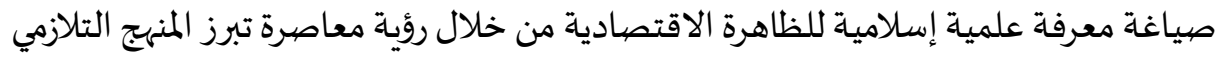

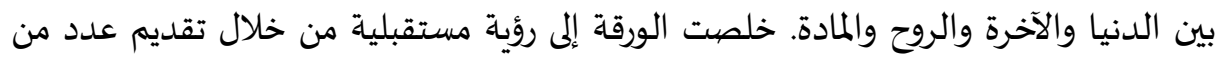

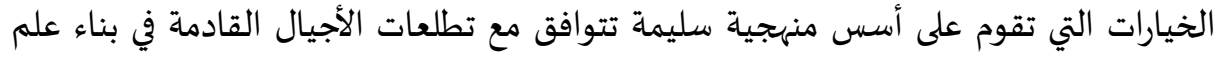
اقتصاد إسلامي يلبي حاجة المجتمعات المعاصرة. الكلمات الدَّالة: المنهجية، الاقتصاد الإسلامي، المراجعة، الاقتصاد التقليدي، الخطاب الاقتصادي السائد. - (المات تصنيف B410, B590:JEL تصنيف H21 
2009م) فما هي انعكاسات تلك المراجعات على

الاقتصاد الإسلامي؟ بمعنى هل من المهم الالتفات إلهها

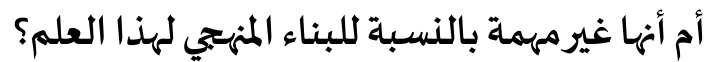

صياغة معرفة علمية إسلامية للظاهرة الاقتصادية، يتطلب تقديم منهجية معاصرة تعكس رؤية المنهجية الإسلامية؛ وتبرز المنهج التلازمي بين الدنيا والآخرة والروح

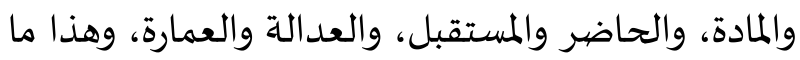
يميز الاقتصاد الإسلامي، لذا من الأفضل التركيز عليه. خامسًا: هل هناك علاقة بين المنهجية التي سار علهيا الاقتصهاد الإسلامي ومعالجة القضايا الملحة التي تعاني

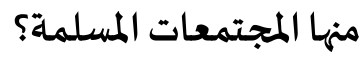

سار الاقتصاد الإسلامي في طريق عرض نظريات الاقتصاد الرأسمالي نفسها، مما أغلق الطريق أمام معالجة الطاقيات

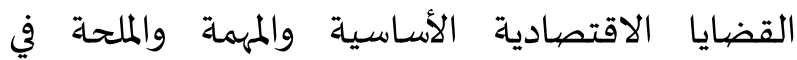

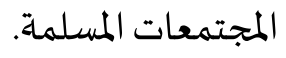

سادسًا: ما أثر المنهجية الأحادية التي سارعها الاقتصاد التقليدي في بناء علم الاقتصهاد الإسلامي في العقود الماضية؟ وهل هناك حاجة لمراجعة ذلك؟ وما السبيل والمخرج الصحيح في هذا البناء؟ وهل يمثل المنهج البيئي

(المخرج المناسب؟ (interdisciplinary approach) أدى منهج تأصيل علم الاقتصاد المعاصر في مجال الاقتصاد الإسلامي إلى تأثيرات عديدة نذكر منها:

$$
\text { التأثير الأول: الوقوع في فخ تأصيل الربا }
$$
يرتكز علم الاقتصاد الرأسمالي على أدوار الربا (سعر الرع الفائدة) في تحريك الأموال من الأغنياء (الأنانية) إلى الى الى الراديا النشاط الاقتصادي من أجل التوازن بين الادخار

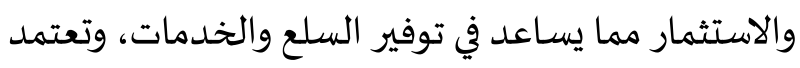
السياسات الاقتصادية عليه في إصلاح اختلال التوازن،
بعد الاطلاع على ورقة عبد الرزاق بلعباس: المفارقة المنهجية في الخطاب الاقتصادي السائد. تنحصر المساهمة التي أقدمها في النقاط التالية: أولاً: كيف ومتى كانت انطلاقة البناء المنهجي للاقتصهاد

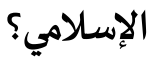
في تقديري أن بداية انطلاقة البناء المنهجي للاقتصاد

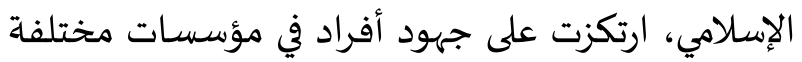

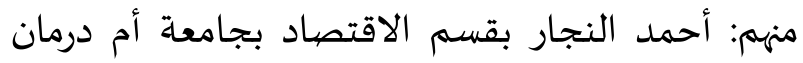
الإسلامية، ومحمد نجاة الله صديقي ومحمد أنس الزرقا بمعهد الاقتصاد الإسلامي بجامعة الملك عبد العزيز، المجاديا

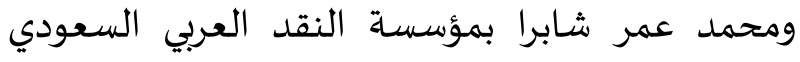
وغيرهم، وكان ذلك بعد الستينات من القرن الماضي. ثانيًا: على أي و/أو أسس بمنهجية بنيت تلك الانطلاقة؟ وهل ارتكزت على منطلقات مناسبة ومعقولة في ظل الظروف التي نشأت في كنفها؟

بنيت المنهجية في مرحلة الانطلاقة على حسب رؤية الباحث دون دور واضيح للمؤسسات، وما تزال تقوم على ذلك، ولعدم رغبة كثير من الباحثين في تكوين منهجية خاصة بالاقتصاد الإسلامي، مازالت المنهجية تعاني من

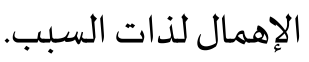
ثالثًا: هل هناك حاجة لمراجعة تلك الانطلاقة؟ أم أنها

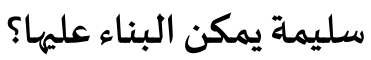
توجد حاجة ضرورية لمراجعة انطلاقة البناء المنهجي

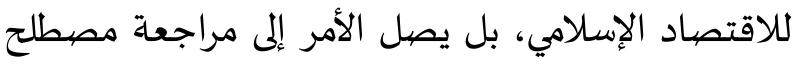
الاقتصاد الإسلامي نفسها.

رابعًا: يشهد الاقتصاد التقليدي (mainstream) (economics مراجعات ومناقشات مهمة منذ أمد بعيد

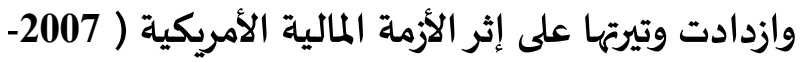


التأثير الثاني: استخدام الأسلوب الكمي للهروب من صياغة نظرية الإسلام الاقتصادية: أدى غياب وضبابية الاقتصاد الإسلامي خاصية في مجال النظرية الاقتصادية، إلى وجود نزعة لدي الباحثين

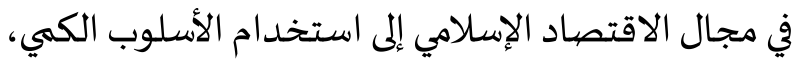
وكثر استخدام أساليب الإحصاء والرياضيات والاقتصياد

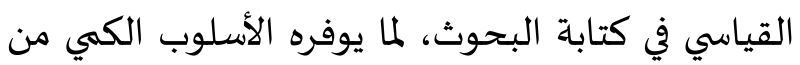
تحديد متغيرات الدراسة المراد تحليلها، مما فتح الباب الباب البان لهروب الباحثين من التفكير في صياغة نظريات الاقتصاد الإسلامي، وترتب على ذلك النتائج الآتية:

1- بعد أن دخل رواد الاقتصاد الإسلامي المعاصر في حيرة تأصيل علم الاقتصاد في صورته الوصفية، تم اعادة إنتاج الأزمة المعرفية في قضايا الاقتصاد القياسي من أجل الحتصاد

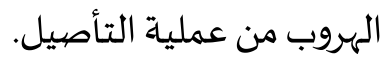

2-سعي أقسام الاقتصاد الإسلاهي إلى ادخال الاقتصاد القياسي في برامج الدراسات العليا، لأن عدم تدريسها يؤدي لقلة فرص تعين واستيعاب طابها حتى في مجال المؤسسات الاقتصادية الإسلامية! التي ينافسهم فهيا طلاب الاقتصاد! 3- البعد عن فهم الترابط الفكري والرياضي في الاقتصاد الإسلامي الذي يظهر في فهم ودراسة العهد النبوي مثال ذلك: عدم تدريس اقتصاديات الميراث وهي مادة تعكس هذا الترابط في أقسام الاقتصاد الإسلامي في كليات الاقتصاد والتجارة (الذين لهم خلفيات رياضية تكتسب في دراستهم)، وتدريسها لطلاب كليات الشريعة (الذين يتم حرمانهم في الدراسة من المهارات الرياضية). 4- البعد عن مصادر التراث واللغة العربية والاقتراب

من مراجع الاقتصياد القياسي.
وبالتالي لا يمكن تصور الاقتصاد الرأسمالي بدون الربا (سعر الفائدة)، ويؤثر على تقييم واختيار المشاريع وأحد العوامل المحددة لكل من الاستهلاك والادخار والاستثمار وغيرها من العوامل الاقتصادية المهمة. يحرم الإسلام التعامل نهائيًا بالربا (سعر الفائدة) من خلال حفظ مقصد المال من جانب العدم، ويأمر بدفع الزكاة في جانب حفظ مقصد المال في جانب الوجود!!؟ وهذا يتعارض مع أصول الاقتصاد الرأسمالي، وبالتالي السير في أسلمه الاقتصاد الرأسمالي قد أفرزت الآتي: 1 - التحايل في إدخال الربا (سعر الفائدة) في الاقتصاد الإسلامي، ومثال ذلك أن من تطبيقات الربا استخدام معدل الفائدة جائز في التحليل في كل من (البيع الآجل - الإجارة الجعالة - المناقصات والمزايدات - تقويم المشروعات - الزكاة - التأمين - الوصايا__توزيع الأرباح وغيرها). 2 - محاولات أسلمه المشتقات المالية وهي تعتمد على الربا. 3 - معاملة الزكاة مثل معاملة الضرائب، مع الفرق

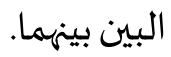
4 - إهمال الحديث عن توازن الاقتصاد الإسلامي في مستويات الكفاف والكفاية والغنى وتحريم حد الرغد، لإنال لعدم معرفة الاقتصاد الدهري لذلك، وبالتالي إدخال التوازن في الاقتصاد الدهري في التحليل الاقتصادي الإسلامي مع استبدال سعر الفائدة بمعدل الريح! 0 5 - الفرح بحديث بعض علماء الاقتصاد الرأسمالي بالوصول بسعر الفائدة بالصفر أو بالسالب، مع النسيان أن منطلقهم هو الدين المسيحي، وليس اعتقادهم في

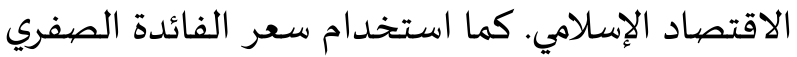
هو مرحلة نادرة في أصول الاقتصاد الرأسمالي. 
1- تطويع الآيات القرآنية الكريمة والأحاديث النبوية

الشريفة والنصوص لتاكيد تطابق ذلك مع النظريات

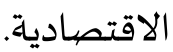

2- صعوبة تأصيل علم الاقتصاد المعاصر، ويرجع ذلك إلى اختلاف مصدر المعرفة بين الاقتصاد المعاصر (العقل

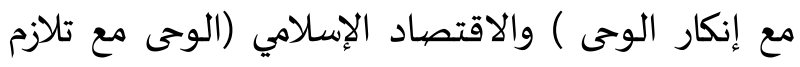

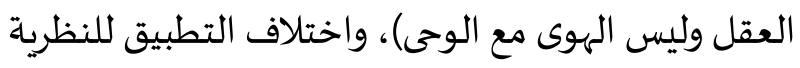

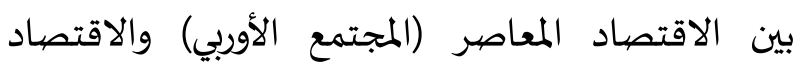

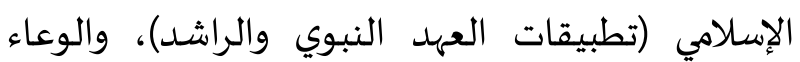
المفسر بين الاقتصاد المعاصر (اللغة الاوربية) والاقتصاد

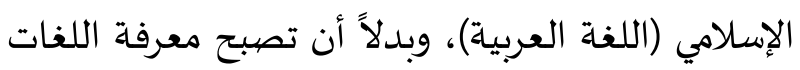

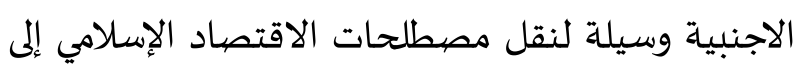
العالم، صارت أداة لإدخال مصطلحات الاقتصاد المعاصر إلى الاقتصاد الإسلامي. 3- انتقال المنطلقات الفكرية لعلم الاقتصاد المعاصر من خرافة المعتقد الديني والتحرر من كافة قيود الحرية كالدين والأسرة وتبرير دوافع الأنانية والبخل والنفعية في سلوك المستهلك والمنتج الى صياغة نظريات الاقتصاد

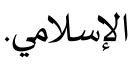

4- تدريس الاقتصاد الإسلامي في الجامعات وفي مواضيع وتعريف ومنهج الاقتصياد المعاصر نفسه. التأثير الر ابع: ظاهرة الإحلال العلمي لأقسام الاقتصهاد

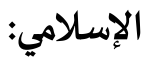
بعيدًا عن العاطفة الدينية نجد أن الموجود من أنواع المعرفة الاقتصادية الإسلامية في أقسام الاقتصاد

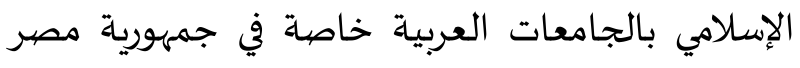

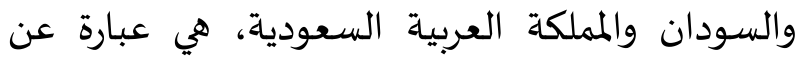
نظريات ومفاهيم علم الاقتصهاد الوضعي، كمدخل ثابت
وضح من السرد السابق أن علم الاقتصاد الرأسمالي،

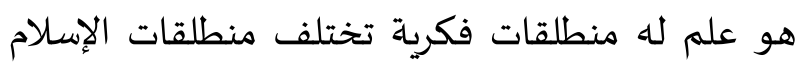

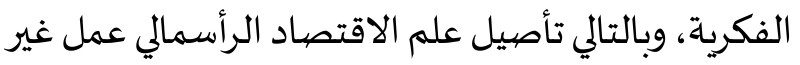
ذي جدوى وأهمية، ولا يساهم في تطوير علم الاقتصاد الإسلامي، بل إن كلمة اقتصاد أصبحت تعنى مصطيح علمي معين. ومن الأمانة العلمية التفكير في مصطلح بديل يعكس رؤية الإسلام الاقتصادية.

التأثير الثالث: تأثير ترجمة مصطلح economics على

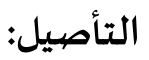

تتمثل مشكلة مصطلح الاقتصاد الإسلامي في أنه كلمة مركبة من اقتصاد وترجح إلي ترجمة مصطلح (economics) التي ترجع إلي نوعين من الترجمة الأولى: علم تدبير المنزل، التهادئ الثانية: علم الاقتصاد.

منذ بداية ظهور مصطلح الاقتصاد الإسلامي في رسائل الدكتوراه في القانون بالجامعات الأوربية، وتدريسه في جامعات العالم الإسلامي، وإدراجه في مواضيع مؤتمرات الإنات الإندات الاقتصاد الإسلامي، ونشر بحوثه في المجلات العلمية،

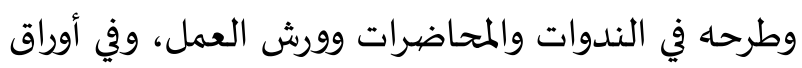

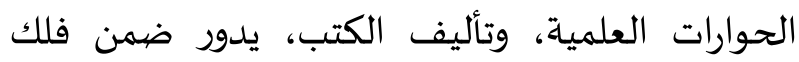
الاقتصاد الرأسمالي. ومن ذلك الزمان الصان أصبح مصطيح الاقتصاد الإسلامي يعاني من عدم استقرار على دلالات

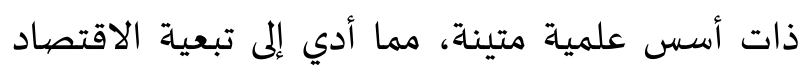
الإسلامي لعلم الاقتصاد الوضعي:

صار علم الاقتصاد الإسلامي تابع لعلم الاقتصاد،

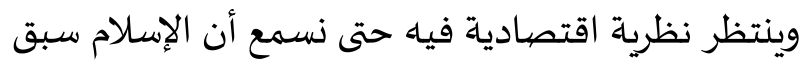

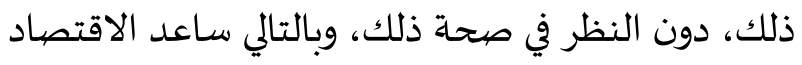
الإسلامي في نشر علم الاقتصاد على حساب الاقتصاد

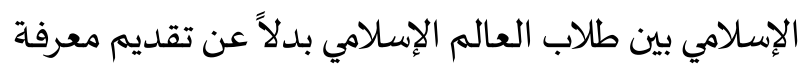
اقتصادية إسلامية حقيقة، ومن أبرز ذلك: 
يتميز النشاط الاقتصادي في الإسلام بالتركيز على البعد الأخلاقي، مما يتطلب انعكاس على السلوك الاقتصادي، وبالتالي تركيز المنهجية على البعد الأخلاقي. ثامنًا: ما هو المستقبل وماهي الرؤية التي يمكن رسمها من هذا المشهد والتي يمكن أن تفيد الأجيال القادمة في البناء

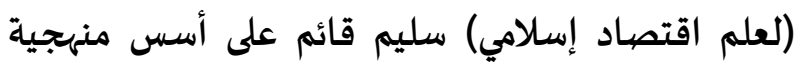
متينة ومستجيب لتطلعات المجتمعات الحديثة؟ عليم المان يصبح المستقبل أمام الاقتصاد الإسلامي يتحمل

$$
\text { الخيارات التالية: - (المبتع }
$$

الخيار الأول: التأصيل الحالي (تأصيل الاقتصهاد الرأسمالي)، وبالتالي تظل الآثار السالبة الحالية لمسيرة الاقتصاد الإسلامي مستمرة.

الخيار الثاني: تأصيل الدمج (تعديل الاقتصاد

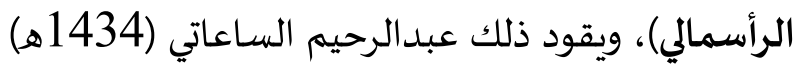

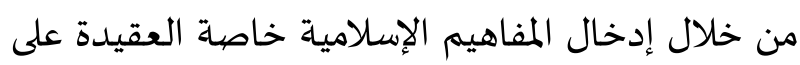

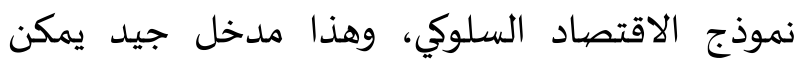
الاستفادة منه في تطوير عملية التأصيل. الخيار الثالث: الصياغة الإسلامية للظاهرة الاقتصهادية: في تقديري تركز هذه المنهجية على الرؤية التالية: 1- التفرقة بين الظاهرة والمعرفة العلمية في الاقتصاد يساهم في التوصل إلى القواعد المنهجية لتأصيل الظاهرة

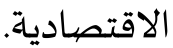

2- يساعد فهم تطبيقات العهد النبوي الاقتصادية في التوصل إلى القواعد المنهجية لتأصيل الظاهرة الاقتصادية. 3- تمهد القواعد المنهجية لتأصيل الظاهرة الاقتصادية لصياغة نظرية اقتصادية إسلامية جديدة. بناء النظرية الاقتصادية الإسلامية يحتاج إلى الاختيار

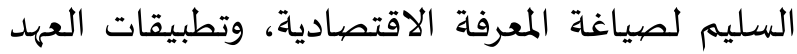
النبوي هي الأنسب لأنها تحتوى على المكونات العلمية
فهها، ثم إحلال اقتصادي يتمثل في ذكر آيات قرآنية كريمة وأحاديث نبوية شريفة تؤكد النظريات والمفاهيم السابقة (الأزهر وأم درمان ومعهد الاقتصاد الإسلامي بجدة والإمام محمد) أو إحلال فقهى يركز على سرد تفرد الشربعة الإسلامية عن الاقتصاد الوضعي (أم القرى واليرموك

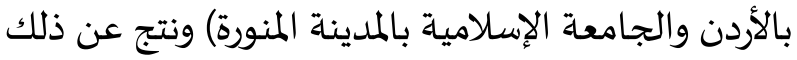

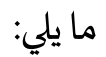
1- عدم المزج الجيد بين العلوم الشرعية والاقتصادية أوجد لنا شخصاُ ذا ثقافتين منفصلتين، اقتصادية

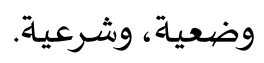

2- المساهمة في نشر علم الاقتصاد الوضعي في أقسام الاقتصاد الإسلامي بكفاءة عالية خدمته أكثر من الاقتصاد الإسلامي. 3- عدم الاستفادة من المنهجية والأسلوب الذي تطورت به العلوم الإسلامية مثل الفقه وأصهول الفقه والقضاء وغيرها، الأمر الذي مكنها من تكوين شخصيتها المستقلة واختلافها الواضح من علوم القانون والفلسفة الترية الدياء

$$
\text { والمنطق. }
$$

4- محاولات تحليل التعامل بالربا (سعر الفائدة) بدلاً عن تفعيل أدوات التمويل والاستثمار والدعم الإسلامي،

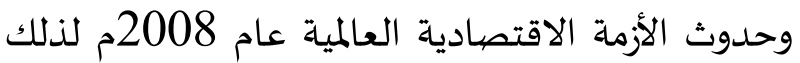
أصبحت التفرقة بين التمويل الربوي والإسلامي أمرًا عسيرًا. سابعًا: هل هناك حاجة للتركيز على البعد الأخلاقي في

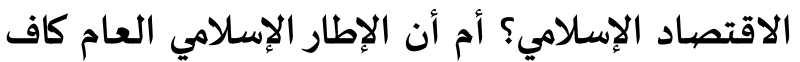
لذلك؟ 
جوانب عديدة في تطوير علم الرياضيات المعاصر عندما

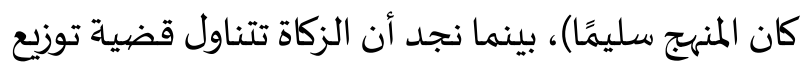

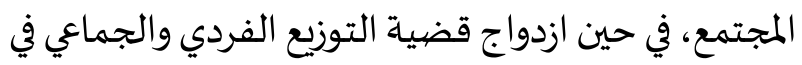
الوقف.

4- يمثل الوضع السـابق الإطار الأسـاسي لعلم المعاش،

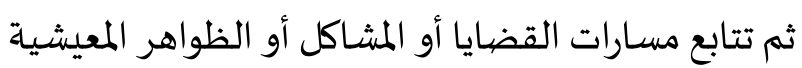
عبر الزمن حتى الوقت المعاصر والدراسة التحليلية لذلك.

5- الدراسة التحليلية تجعل لنا المقدرة على استخلاص الأفكار الاقتصادية التي تصلح للعرض كنظريات اقتصادية في بطون أمهات الكتب خلال الأربعة عشر قرنًا

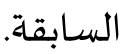

6- النتيجة السابقة نكملها بالقدرة على تتبع التطور

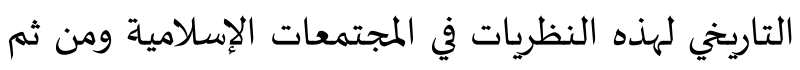
معرفة أسباب فناء ما فني منها وما لقيا من تطور وترقية على أيدي مؤيديها حتى نقف على آخر وأكمل صورها. يتطلب الأمر دراسة النسق المعرفي الإسلامي، يبين لنا أن صياغة الإسلام العلمي لدراسـة الظاهرة الاقتصادية، ومن ثم وضع النظريات، قد مر بالمراحل التالية: (المرحلة الأولى: مرحلة علوم الوحي، وفيها يمكن صياغة النظرية الاقتصادية من خلال الآيات القرآنية التي تناولت الظاهرة الاقتصادية، ونصوص الأحاديث النبوية الشريفة في المجال الاقتصيادي نفسـه، وكانت هذه المرحلة منذ بداية القرن الهجري الأول وحتى بداية القرن الهجري الثاني)(

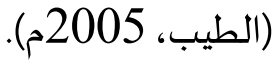
شكلت المرحلة فترة تدوين المعرفة الإسلامية التي

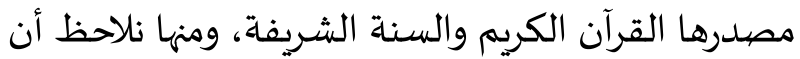
من قام بالتوثيق والتدوين، أغلبهم كانوا من صغنار

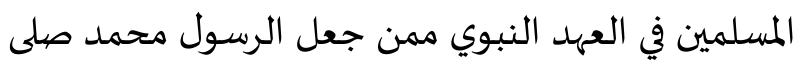

والعملية للاقتصاد الإسلامي، وفيها تكامل مكونات المعرفة وهي : مصدرها والمتمثل في الوحي، وبيئة المعرفة وتعتبر فترة العهد النبوي أفضلها، والتطبيق الأمثل، ومن هذه الفترة يمكن استنباط ملامح النظرية. يعتمد منهج صياغة المعرفة الاقتصادية من تطبيقات اسطرئه العهد النبوي على منهجية تختلف عن علم الاقتصياد الرأسمالي المعاصر، وعلم الفقه، وهي منهجية مختية مختلفة تحتية خاصةة باء، وهي ترتكز على الآتي: 1- تناول القضية أو المشكلة أو الظاهرة المعيشية من

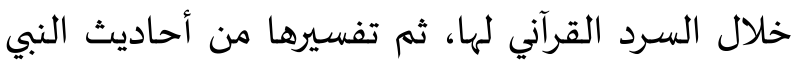
محمد صلى الله علياه وسلم. 2- معرفة تطبيقاتها في العهد النبوي أولاً من تتابع

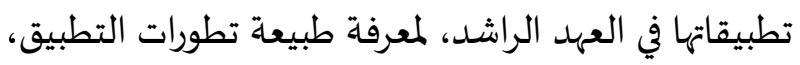
وتحديد جوانب الاتفاق والاختلاف مع تطبيقات العهد لمدربه النبوي.

3- استعمال الترابط الفكري والرياضي لها، ودمج النظرية والتطبيق، وهذا الأسلوب يبقي واضحًا في علم المعاش وفي علم الفرائض، وهو يتناول قضية توزيع ميراث فرد في الأسرة من منظور فكري ورياضي ونظري وتطبيقي، تتداخل فيه قضايا الإطار الفقهي وحفظ كليات الشريعة مرية

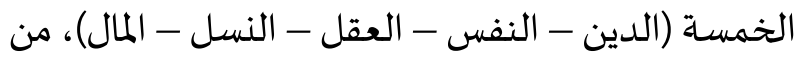
خلال أركان وأسباب وموانع الإرث وحدود تصرف المورث في حياته على الوصية والوقف، بمنهجية تدخل الأرقام والرياضيات، دون أن يشعر الطالب في كليات الشريعة (معظم طلاب الشريعة في الوطن العربي ليس لهم اهتمام

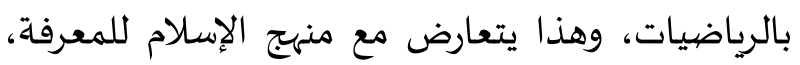

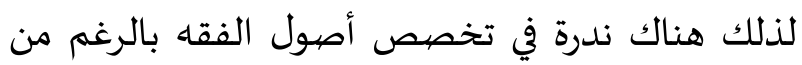
أهميته في تطوير علوم الشريعة) بوجود تعارض في المادة، ويرجع صعوبة المادة إلي اهمال تدريس الرياضيات (هذا

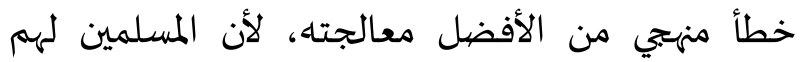


للتدليل على دور تتابع النسق المعرفي الإسلامي في صياغة النظريات، نأخذ كتاب مقدمة أبن خلدون، (ففكرة تقسيم الملك إلى ثلاثة ذكرها ابن المقنع، وأسباب

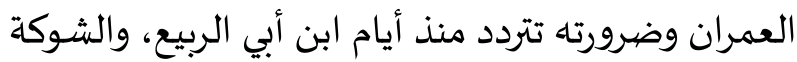
أو العصبية وتكوين الدول مذكور عند الجويني والغزالي. والعمران وعلاقته بالعدل والظلم ذكرها ابن رضوان. ) ) (عارف،1994 م). ويترب على هذه القاعدة ضرورة صياغة النظرية الاقتصادية من خلال:

1- ربط الظاهرة الاقتصادية بالقواعد الشرعية والاجتماع والسياسة كمنهج أصيل.

2- (فهم حدود العلاقة بين الفكر والواقع الذي برز فيه فهمًا يؤدي إلى عمل منهجي مستقيم مع هذا الفكر، بحيث لا يؤدي إلى طمره في واقعه التاريخي، ومن ثم يلغي إمكانية الاستفادة منه وإبراز ما يتجاوز الزمان والمكان في أفكاره وفي الوقت نفسه لا يضفي عليه صفة العموم والإطلاق

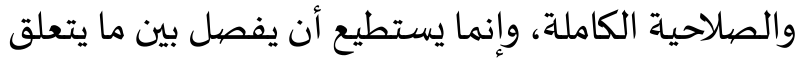
بمعطيات الزمان والمكان وخصوصية الإنسان، وبين ما يتعلق بالنسق المعرفي الإسلامي ) (عارف، 1994م). 3 - التفرقة بين الفقه الاقتصادي والاقتصاد الإسلامي

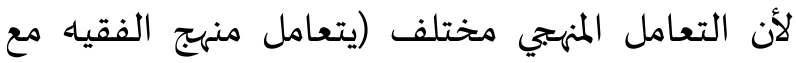
النص بصورة تفوق تعامله مع الواقع، فهو منهج لتحليل النص وتفسيره في ظل إدارة الواقع وتحقيق مناطه وتحديده وتخريجاه مما يمكن إنزال الحكم المناسب عليه) (عارف، 1994 م )، أما منهج الاقتصادي فيعتمد على إنى فهم الواقع الذي يحيط بالظاهرة الاقتصادية وتفسيره استنادًا على فهم النص، ومثال ذلك تعدد رؤية المدارس الفقهية في المعاملات الاقتصادية.
الله عليه وسلم، يجعل تعليم القراءة والكتابة، شرطًا لفك

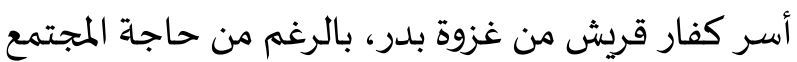
الإسلامي للمال، وبالتالي نستنتج أن الاقتصاد الإسلامي يركز على التعليم قبل التمويل. (المرحلة الثانية: مرحلة الفقه الاقتصادي، وذلك منذ النذالئ بداية القرن الهجري الثاني وحتى القرن الثالث، وفيها ظهرت

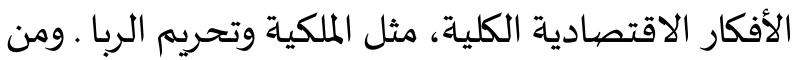
أبرز كتب الفقه الاقتصادي في تلك المرحلة: بداية المجتهد

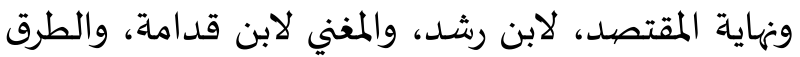
الحكيمة في السياسة الشرعية لابن القيم الجوزية وغيرها)) (الطيب، 2005م). المرحلة الثالثة: مرحلة الدراسات الاقتصادية المستقلة، ظهرت في الفترة من القرن الثالث الهجري وحتى القرن السابع الهجري، ومن أبرزها، كتاب الخراج للقاضي يعقوب الفرن الفرت الفرن

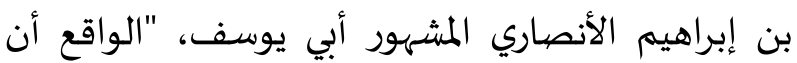

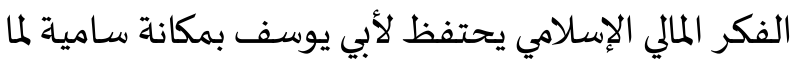
قدمه له من عطاء مبكر وعميق من خلال كتابه الخراج،

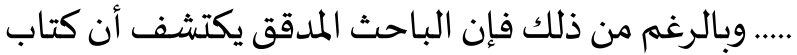
الخراج ليس كتاب في المالية العامة في الإسلام فحسب، وإنما هو أوسع من ذلك، فقد ضمنه الكثير من الأفكار الاقتصادية"، (دنيا، 1984م). المرحلة الرابعة: مرحلة الاقتصاد التحليلي الذي يعتمد

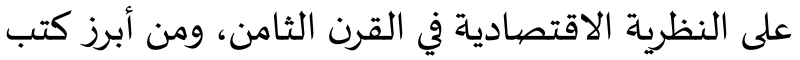

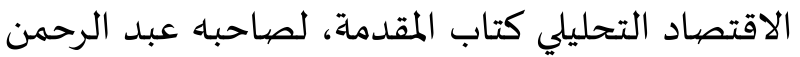
بن خلدون، فقد أفاد العالم الغربي ((بسبقه العلمي في مجال فلسفة التاريخ، كعلم امتد وتم تأصيله وبلورته عندهم، وأفادوا منه في قراءة المجتمعات البشرية، والعوامل الحاكمة للحركة التاريخية، وأنشأوا نواة العلوم الاجتماعية)) ( (عويس، 1996م). 


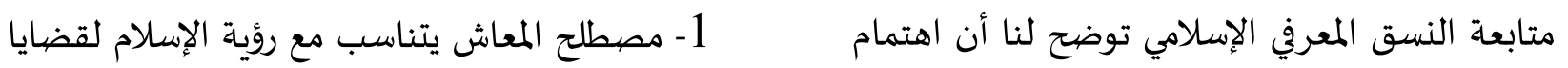
الإسلام بالكتابة في مجال الاقتصاد، سارت حسب تسلسل الاقتصاد.

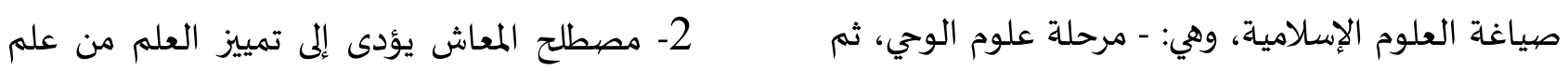
مرحلة علم الفقه الاقتصادي، تلتها مرحلة الدارسات الاقتصاد المعادية المعاصر.

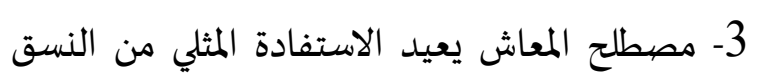
المعرفي الإسلامي في المجال الاقتصادي، وفهم الآيات القرآنية الكريمة والأحاديث النبوية الشريفة، وكافة كتب التراث. 4- يمتاز مصطلح المعاش بأنه يشمل بقية مصطلحات

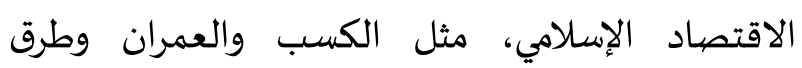
الحصول على الرزق والعمل لكسب قوت الحياة والأعمار

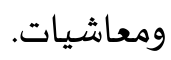

5- إن القاعدة المشهورة (لا مشاحة في الاصطلاح)

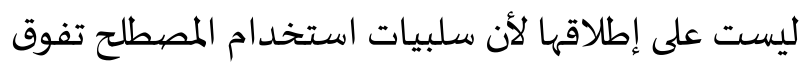

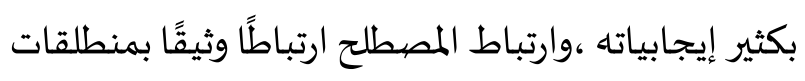
فكرية تخالف رؤية الإسلام. الاقتصادية المستقلة، وأخرها مرحلة الدراسات التحليل الاقتصادي وقد انتهت عند كتاب مقدمة بن خلدون.

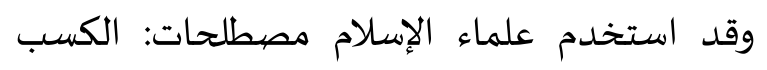
والعمران والمعاش وغيرها، ويمكن اختيار أحدها كبديل

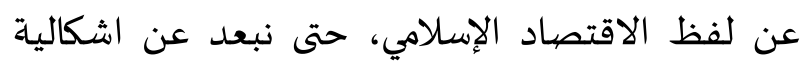
مصطلح الاقتصاد الإسلامي. تناول العلماء المسلمون عملية صياغة النظرية

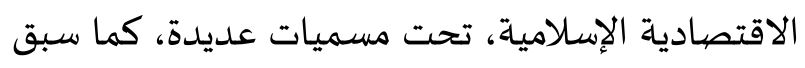
ذكره، ولكن من الفضل استخدام المعاش. أ - مبررات اختيارعلم المعاش كبديل لعلم الاقتصهاد

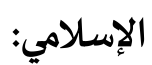

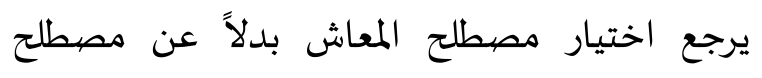
الاقتصاد الإسلامي إلى الأسباب التالية:

عزالدين مالك الطيب محمد، أستاذ الاقتصاد والاقتصاد الإسلامي، قسم الاقتصاد بجامعاة أم الإمادية

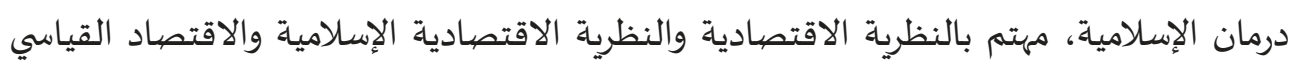

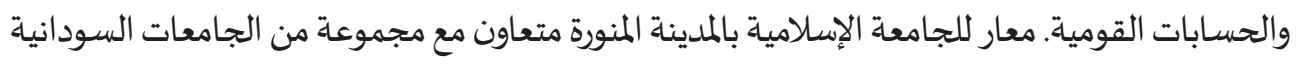
ومراكز أبحاث الاقتصاد والاقتصاد الإسلامي. البريد الإلكتروني: ezzmalik2002@gmail.com 


\title{
Methodology of the Revival of Islamic Economics: A Revisit
}

\author{
Izz El-Din Malik El-Tayeb Mohamd \\ Professor of Economics and Islamic Economics \\ Omdurman Islamic University - Sudan
}

\begin{abstract}
The paper provides concise and focused answers to the questions presented by the editorial board of the journal. These questions revolve around the start of the systematic construction of the Islamic economy and its foundations. The paper emphasizes the importance of reviewing this breakthrough but goes further. It draws attention to the importance of revising the term Islamic economy, based on the revisions of the traditional economy currently considering the repercussions of the US financial crisis (2007-2009). The paper justifies the importance of revision to reach the formulation of Islamic scientific knowledge of the economic phenomenon through a contemporary vision highlighting the synergistic approach between the world and the hereafter, spirit and matter. The paper concludes with a vision for the future by presenting several options based on sound methodologies that are consistent with the aspirations of future generations in building an Islamic economics that meets the needs of contemporary societies.
\end{abstract}

KEYWORDS: Methodology, Islamic Economics, revival, conventional economics, mainstream economics discourse.

JEL Classification: B410, B590

KAUJIE Classification: $\mathrm{H} 21$ 